\title{
Study on Microstructural Changes of Coal after Methane Adsorption
}

\author{
Feng Zengchao ${ }^{1}$, Zhou Dong ${ }^{1}$, Zhao Yangsheng ${ }^{1}$, Cai Tingting ${ }^{1}$ \\ ${ }^{1}$ Mining Technology Institute, Taiyuan University of Technology, Taiyuan, Shanxi 030024
}

\begin{abstract}
This study analyzes the microstructure and deformation rule after methane adsorption on coal by scanning electron microscopy (SEM) and computed tomography (CT) scanning of microscopic coal samples. Studies have shown that coal is a natural rock composed of vitrinite coal matrix and clay mineral. After methane adsorption, coal undergoes non-uniform expansion deformation. This occurrence prompts coal density to decrease and then increase, causing the density distribution of coal to become highly concentrated. During swelling after adsorption, the effects of deformation and expansion on coal structures become stronger than that of mutual squeezing. Under low adsorption pressure, coal expansion deformation are more likely to crack the pore structure of the original coal to acquire space for expansion. When the adsorption pressure increases, compression becomes mainly concentrated in the low-density region; as adsorption pressure continues to increase, expansion deformation occur from high-density to low-density regions. The methane adsorption properties of coal are related to its pore structure. Adsorption and swelling mainly occur in the region where the pore structure is unfilled or filled with clay minerals. Expansion deformation conforms to the Langmuir equation; the region without pore structure development exhibits no swelling; the deformation degree and range of the pore structure and clay mineral mixing zone exhibit increase volatility. Overall, results reveal a microstructural change after methane adsorption.
\end{abstract}

Keywords: scanning electron microscopy; computed tomography; swelling and deformation; adsorption; pore structure

\section{Introduction}

Coal and methane gas adsorption involves physical adsorption, causing expansion deformation of coal and decreases in elastic modulus and mechanical strength after methane adsorption. A higher adsorption pressure indicates more obvious occurrence of such phenomenon; under external constraints, coal also generates additional stress and reduces the permeability of coal after methane adsorption (Wang et al., 1996; Lin et al., 1986; Zhao et al., 2009). Coalbed methane desorption stages were divided by $\operatorname{Meng}(2014)$ in basis of desorption velocity. It was showed that the reason why causing desorption velocity changed were not only the diversification of gas in coalbed pressure, but also the contraction of coal matrix after desorption. Coal is a naturally porous medium, and its physical properties in methane adsorption are related to the microscopic pore fissure structure and coal rock components (He et al., 1996; $\mathrm{Wu}$ and Zhao, 2005). However, to evaluate the physical changes in coal after methane adsorption, constant adsorption pressure must be applied. Most of these changes are evaluated through a macro-experiment. From a mesoscopic point of view, the internal structure of coal undergoes deformation after methane adsorption. The mechanism and factors underlying these changes need to be examined by mesoscopic observations.

Scanning electron microscopy (SEM) uses secondary electron signal imaging to observe the surface morphology of the sample to infer the material composition and reveal the mesoscopic structure on the material surface (Zhang and $\mathrm{Li}, 2004$ ) ; CT scanning is a nondestructive experimental procedure used to detect the internal structure of a material by the X-ray absorption difference of materials exhibiting different densities. CT scanning can identify the physical parameters of materials (Li et al., 1999; Feng, 2008). These two techniques provide ways to detect the mesoscopic structure of a material. SEM is employed to examine the pore characteristics of coal, including coal pore fissure, mineral composition, and microstructure (Zhang and Li, 2004; Cetine, 2004; Rameshks, 2004). Karacan C.O (2001) et al. combined X-ray CT and SEM to observe and evaluate gas migration and adsorption ratio difference, as well 
as different types of microstructures in coal. However, the mechanism of coal expansion deformation after methane adsorption, as well as the damage rule of the mesoscopic structure have not been investigated because of limitations in research methods. The present study used SEM technology and micro-CT to evaluate the effect of methane adsorption on the mesoscopic structure of coal. From the research of this paper, it is not only illustrated the structural changes, but also formed a basis of non-uniform diversification of permeability after methane adsorption.

\section{Experiment}

\subsection{Coal sample preparation}

The coal samples used in the experiment were collected from the Sijiazhuang coal mine of Yangmei Group. The coal used was anthracite, and a mesoscopic coal sample was obtained using a manual drill measuring $15 \mathrm{~mm}$ in height and $8.5 \mathrm{~mm}$ in diameter. By using a precision cutting machine, the coal sample was cut in half along the axis and used as backup after cleaning and drying.

\subsection{Scanning electron microscopy}

SEM was performed using a JSM-7001F thermal field emission scanning electron microscope. During scanning, a top radial cross-section was selected from the prepared coal samples. A target area measuring $1.856 \mathrm{~mm}$ in width and $1.392 \mathrm{~mm}$ in height was selected to set the range for SEM. Scanning was conducted at full width, and the area scanned was magnified under the electron microscope. The scanning voltage was $5 \mathrm{kV}$, and the width was $10 \mathrm{~mm}$. Magnification of a full-width scan was 65 times, and that of the target scanning area was 1040 times.

\subsection{Micro-CT scan}

A CT scanning experiment uses a $\mu \mathrm{CT} 225 \mathrm{kVFCB}-\mathrm{type}$ high-precision micro-CT experiment system (Yu et al., 2010). An experimental system is mainly composed of the following parts: (1) an adsorption chamber made of low-density and high-strength magnesium alloy materials and (2) a precision digital pressure gauge, which is used to display the pressure of methane gas in a specimen chamber. To emit X-ray through the X-ray machine in a microscopic CT system, a specimen device is fixed in the rotation platform of the CT system and then rotated at a constant scanning speed. The turntable angle resolution is set to 655360 steps/rev. The displacement precision of the microscopic CT motion controller is $0.02 \mathrm{~mm}$.

The experimental observation principle is illustrated in Figure 1. Vacuum processing of the coal sample was performed in a specimen chamber. A $2 \mathrm{XZ}-0.5$ type double rotary vane vacuum pump was used to obtain a vacuum degree under $0.6 \mathrm{~Pa}$ in the coal sample. The gas inside the coal sample and the specimen chamber were then discharged. The coal samples under vacuum were scanned, and methane gas $(99.99 \%)$ was injected for another scanning after adsorption equilibrium was reached. The methane adsorption pressure measured 1, 2, 3, 4, and $5 \mathrm{MPa}$, and each time, under adsorption pressure was $12 \mathrm{~h}$ to ensure that the specimen reached adsorption equilibrium. The CT images of the corresponding areas of SEM under different adsorption pressures were then obtained. Data from CT scanning were processed using Matlab.

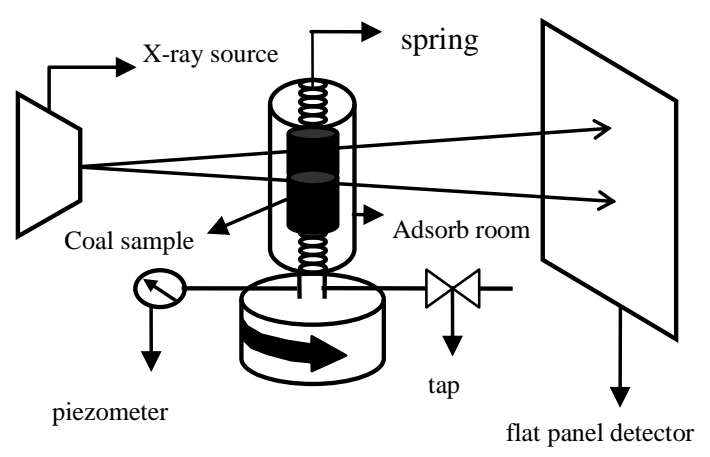

Fig.1 Microscopic CT observation of experimental elementary diagram of methane adsorption coal

\section{Coal body structure analyses by SEM and CT scanning}

\subsection{Scanning electron microscopy}

Electron microscope scanning results are shown in Fig. 2. The diagram shows that the pore fracture morphology of the targeted coal surface is clearly visible and can determine the components of coal. The 
main components of the full-width scanning area comprised vitrinite coal matrix, which contained mottled or stripped clay minerals filling areas of different sizes. The length and width of the full-width scanning area were divided into 16 equal parts to obtain 256 pieces of target areas of the same size. Further microscopic information on the target areas can be obtained by magnification and target scan. In accordance with the degree of pore fracture development and its filling condition, the target areas of coal can be divided into four categories: no-development pore area (A1, A2, A3), not filled pore area (B1, B2), mineral-filled pore structure area $(\mathrm{C} 1, \mathrm{C} 2)$, and mixing area of pore fissure structure and clay minerals (D1, D2).
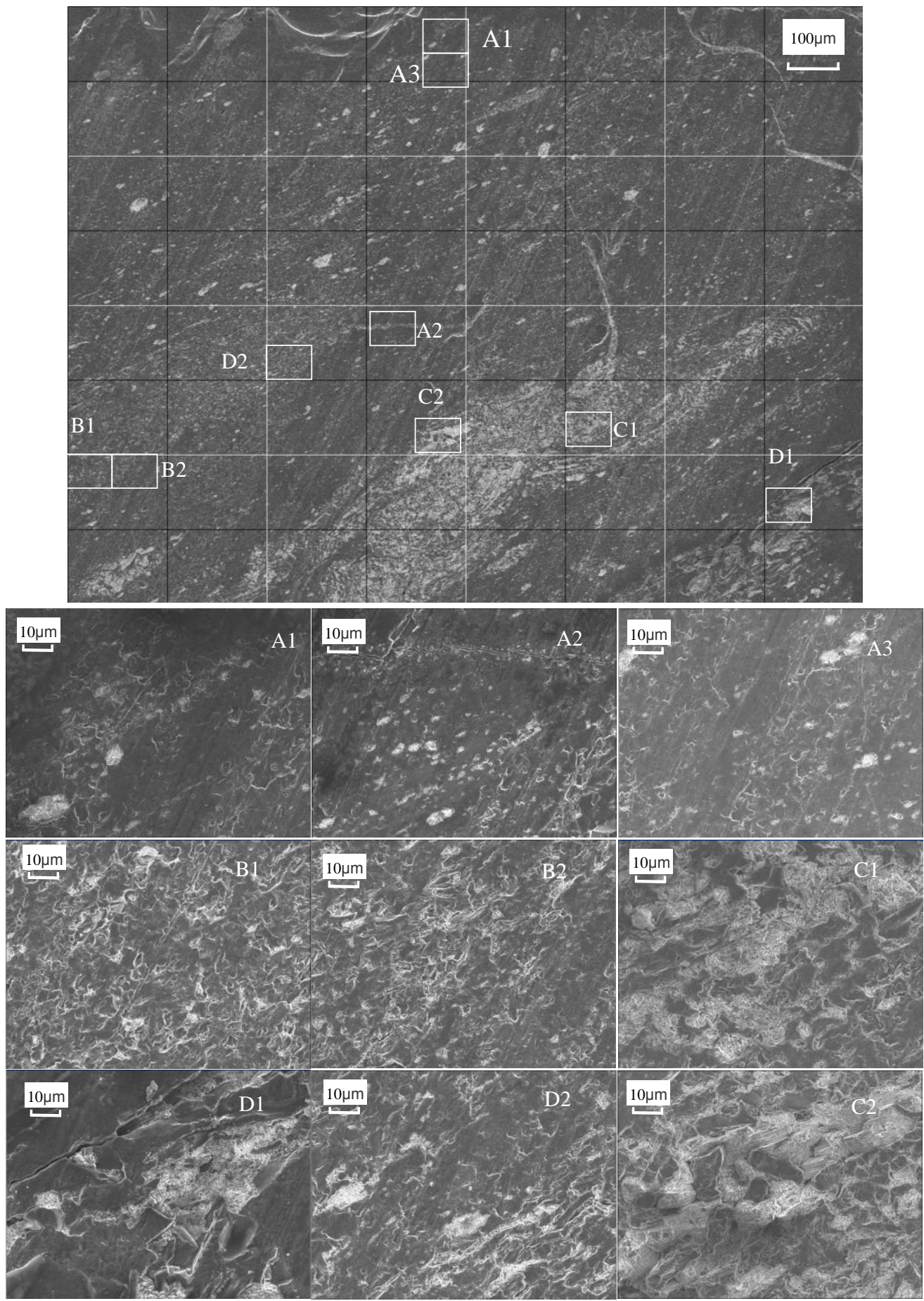

Fig.2 SEM results and pore structure classification 
section and obtain the section image of the change in material density. Attenuation coefficient is the strength variation when the ray passes through the random point of the object cross-section. The relationship between the coefficient and density of the object is expressed as follows (ASTM E1935-97):

$$
\mu_{i, j}=\mu_{m} \rho_{i, j}
$$

where $\mu_{m}$ is the mass attenuation coefficient, and $\rho_{i, j}$ denotes the density of an object point to be evaluated. The attenuation coefficient array of the cross-section is obtained and converted to grayscale to obtain the real information of the internal structure of the coal body. On the basis of the density change in coal, the present study hypothesized the pore structure changes after methane adsorption and a change in deformation trend.

A full-width scanning area image obtained by CT scan is shown in Fig. 3. The whole scanning area measures $320 \times 240$ pixels, and the width of each CT pixel is $5.8 \mu \mathrm{m}$. To analyze the characteristics of the target area of the coal rock structure under microscopic CT, the length and width of this area are divided into 16 equal parts. Grid partition is conducted to obtain 300 target areas $(20 \times 15$ pixels $)$. The average attenuation coefficient of each target area is calculated. The area proportion in each attenuation coefficient section is counted with 0.005 intervals to obtain the attenuation coefficient distribution diagram of the target area unit (Fig. 4).

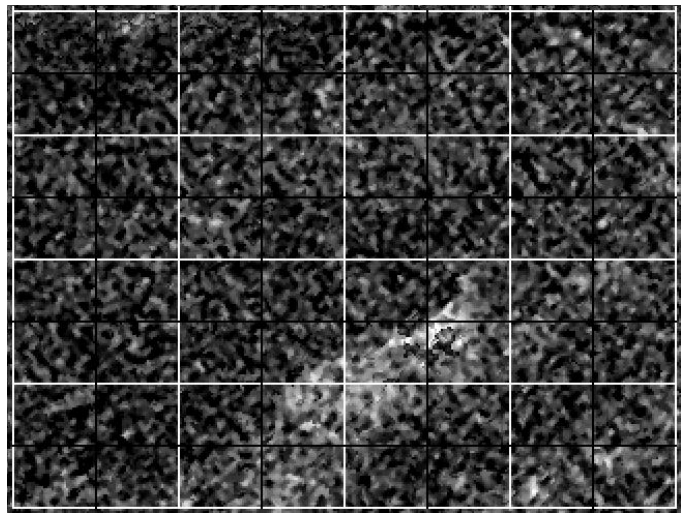

Fig.3 Grayscale of coal samples after CT scanning

As shown in Fig.4, coal is a naturally heterogeneous rock mass. The CT attenuation coefficients of the samples in this area under a vacuum state are distributed between 0.002 and 0.0157 , and the average attenuation coefficient is 0.00477 . The majority of the coal body attenuation coefficients concentrated within a small range. Among them, 89.06\% ranged from 0.02 to 0.06 , thus comprising the majority of the region, and only $10.94 \%$ exceeded 0.06 . Compared with the SEM image, the gray area smaller than 0.06 is composed of vitrinite and part of clay minerals; the bright white clusters or spots greater than 0.06 comprise the pore structures mainly filled with minerals. The density of clay minerals is significantly greater than the density of the coal matrix; thus, the differences in coal body density distribution are mainly attributed to the degree of filling of clay minerals.

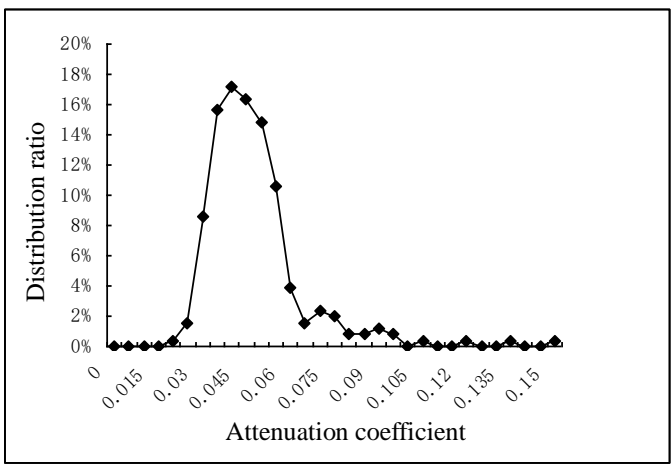

Fig.4 Attenuation coefficient of the distribution ratio of coal samples

\section{Classification of coal microstructure and adsorption characteristics}

Methane adsorption mainly occurs on the coal surface. Methane adsorption capacity and density changes exhibit heterogeneity. Adsorption capacity and structural changes in coal are attributed to varying pore structures.

\section{A. No-development pore area}

As shown in Fig. 2, class A area mainly includes the compact vitrinite coal matrix, and its pore structure demonstrates no development. In this area, few pores are filled with mottled clay minerals. This area type exhibits poor adsorption capacity. After methane injection, the area shows no obviously active expansion deformation. Under the extrusion of the surrounding structure or traction, the attenuation coefficient slightly fluctuates in the 0.001 range. As shown in Fig. 5, A1, A2, and A3 present a maximum motion of $0.00063,0.00131$, and 0.00066 , respectively A2 displays more filled pores; thus, the adsorption pressure increases. When A2 presents a declining 
curve, the fluctuation range reaches the maximum value.

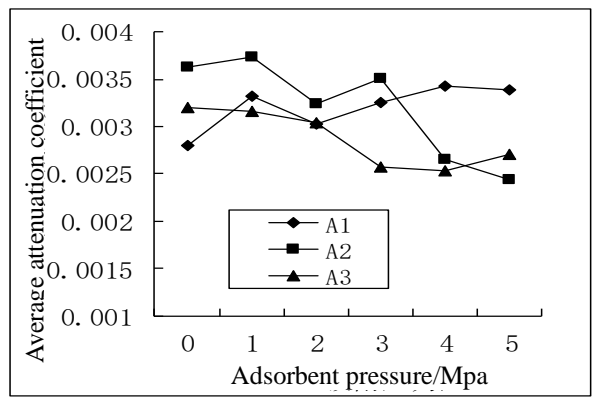

Fig.5 Average attenuation coefficient changing curve of no-development pore area with pore pressure

Statistical results indicate the decreased pixel ratio of the attenuation coefficient in class A area under different adsorption pressures (Table 1). Under different adsorption pressures, the average areas with decreased density in class A area are $41.53 \%, 53.40 \%$, and 50.80\%; the pressure fluctuation is also small under different pressures. The maximum fluctuation of $\mathrm{A} 2$ is $8.67 \%$. A1 exhibits volatility of less than $1 \%$, which shows that the no-development pore structure can remain stable under different adsorption pressures.

\begin{tabular}{cccc}
\multicolumn{2}{c}{ Table 1 Density decreased area ratio of no-development pore area } \\
\hline \multirow{2}{*}{$\begin{array}{c}\text { Adsorbent pressure } \\
\text { /Mpa }\end{array}$} & \multicolumn{3}{c}{ Area code } \\
\cline { 2 - 4 } & $\mathrm{A} 1$ & $\mathrm{~A} 2$ & $\mathrm{~A} 3$ \\
\hline 1 & $42.00 \%$ & $50.67 \%$ & $48.00 \%$ \\
2 & $42.00 \%$ & $50.00 \%$ & $46.67 \%$ \\
3 & $41.33 \%$ & $52.67 \%$ & $51.00 \%$ \\
5 & $40.67 \%$ & $55.00 \%$ & $52.00 \%$ \\
5 & $41.67 \%$ & $58.67 \%$ & $56.33 \%$ \\
\hline
\end{tabular}

\section{B. Unfilled pore area}

Fig. 2 shows the class $B$ area with a highly developed pore structure. Most of them are not filled with mineral holes; a small amount of primary pores less than $5 \mu \mathrm{m}$ in size also exist, and the area presents

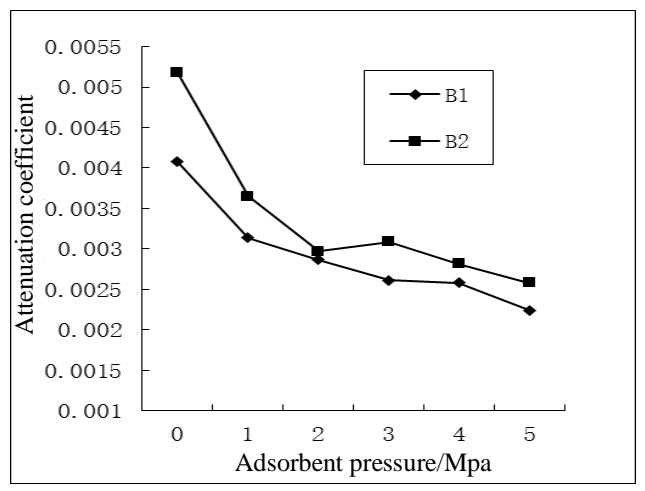

Fig. 6 Changing curve of average attenuation coefficient in the unfilled pore area with pore pressure change a huge surface for methane adsorption. As shown in Fig. 6, the average attenuation coefficient of this area is significantly reduced after methane adsorption; with an increase in adsorption pressure, a marked decline occurs. The maximum change rates that B1 and B2 attenuation coefficient can reach are $44.92 \%$ and $44.92 \%$, respectively, suggesting that relatively obvious expansion deformation occurred in this region, making this the main area where the coal body adsorbs methane, as well as causes expansion deformation.

The statistical results of the density decreased area ratio in Class $\mathrm{B}$ area under different adsorption pressure (Table 2) indicate that the average ratio of density decreased area in class $\mathrm{B}$ area can reach $67.67 \%$ and $67.33 \%$, and the volatility of B1 and B2 is approximately $10 \%$ under different pressures. Additionally, the decrease in density is obvious.

Table 2 Attenuation coefficient decreasing ratio and density

decreased area ratio in the unfilled pore are

\begin{tabular}{ccccc}
\hline \multirow{2}{*}{$\begin{array}{c}\text { Adsorbent } \\
\text { pressure } \\
\text { /MPa }\end{array}$} & \multicolumn{2}{c}{$\begin{array}{c}\text { Decrease rate of } \\
\text { attenuation coefficient }\end{array}$} & \multicolumn{2}{c}{ Reduced area of density } \\
\cline { 2 - 5 } & $\mathrm{B} 1$ & $\mathrm{~B} 2$ & $\mathrm{~B} 1$ & $\mathrm{~B} 2$ \\
\hline 1 & $22.90 \%$ & $29.44 \%$ & $59.67 \%$ & $63.00 \%$ \\
2 & $29.62 \%$ & $42.61 \%$ & $61.00 \%$ & $65.67 \%$ \\
3 & $35.83 \%$ & $40.26 \%$ & $72.67 \%$ & $68.00 \%$ \\
4 & $36.60 \%$ & $45.49 \%$ & $61.33 \%$ & $73.00 \%$ \\
5 & $44.92 \%$ & $50.03 \%$ & $67.67 \%$ & $67.33 \%$ \\
\hline
\end{tabular}

\section{Developed area of the pore structure filled with} minerals

As shown in Fig. 2, the class $\mathrm{C}$ area shows highly developed pore structure, mostly consisting of mineral holes and almost filled with clay minerals. With the increased adsorption pressure, the attenuation coefficient decreases continuously (Fig. 7). The ratio of the maximum change in the attenuation coefficients

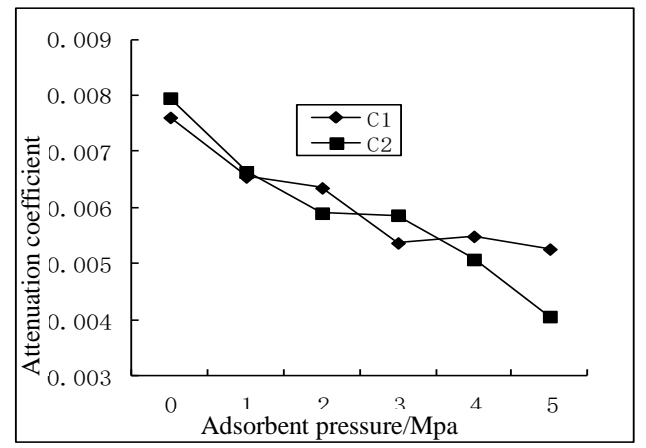

Fig. 7 Changing curve of average attenuation coefficients in the pore area filled with clay minerals with pore pressure change 
of $\mathrm{C} 1$ and C2 can reach $31 \%$ and $49 \%$, respectively. Relatively marked expansion deformation occurred, Table 3 Attenuation coefficient decreasing ratio change in the pore area filled with clay minerals with the change of adsorption pressure

\begin{tabular}{ccccc}
\hline $\begin{array}{c}\text { Adsorbent pressure } \\
\text { /MPa }\end{array}$ & \multicolumn{2}{c}{$\begin{array}{c}\text { Decrease rate of } \\
\text { attenuation coefficient }\end{array}$} & \multicolumn{2}{c}{$\begin{array}{c}\text { Reduced area of } \\
\text { density }\end{array}$} \\
\cline { 2 - 5 } & $\mathrm{C} 1$ & $\mathrm{C} 2$ & $\mathrm{C} 1$ & $\mathrm{C} 2$ \\
\hline 1 & $13.97 \%$ & $16.56 \%$ & $63.67 \%$ & $61.25 \%$ \\
2 & $16.52 \%$ & $25.82 \%$ & $65.67 \%$ & $67.25 \%$ \\
3 & $29.43 \%$ & $26.27 \%$ & $66.00 \%$ & $69.08 \%$ \\
4 & $27.89 \%$ & $36.19 \%$ & $64.67 \%$ & $71.17 \%$ \\
5 & $30.87 \%$ & $49.00 \%$ & $74.67 \%$ & $78.00 \%$ \\
\hline
\end{tabular}

The statistical results of the density decreased area ratio in Class $\mathrm{C}$ area under varying adsorption pressures show that the average ratio of reduced density in class $\mathrm{C}$ area can reach $66.93 \%$ and $69.35 \%$, and the volatility of $\mathrm{C} 1$ and $\mathrm{C} 2$ is more than $11 \%$ and $16.75 \%$ under different pressures. The decreases in density and area are obvious. Studies have shown that clay particles exhibit intergranular pores (Ji et al., 2012). The rich clay rock intergranular pores are very small because the clay particles are also small. The sizes of most of these pores range from tens of nanometers to several micrometers. A more developed micropore presents greater methane adsorption of clay rock. The growth of clay rock micropores controls the

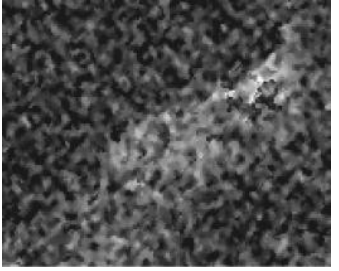

0MPa CT grey scale map
With regard to the adsorption pressure influence on coal body expansion deformation, Pan presented a model of gas adsorption and coal matrix expansion (Pan et. al., 2007), Zhou(1990) studies have determined the expansion properties of coal sample adsorption gas by using resistance strain gauge. When the coal adsorbs gas, the expansion is proportional with the adsorption quantity, and Expansion deformation conforms to the Langmuir equation:

internal surface area of mineral rock, which determines the gas adsorption capacity. Therefore, the clay minerals in coal significantly influence methane adsorption, as well as expansion deformation.

A pseudo-color diagram of density changes under

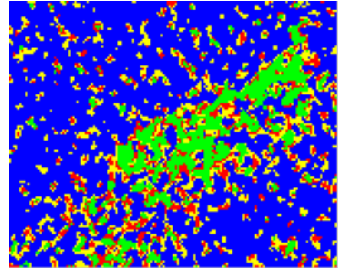

OMPa CT pseudo color map varying adsorption pressures can be obtained. Through the attenuation coefficient range division, CT images of clay mineral area are displayed in different colors, as shown in Fig. 8. The attenuation coefficients of the green, red, yellow, and blue areas are presented as follows: greater than $0.11,0.09-0.11,0.07-0.09$, and less than 0.007, respectively. The observation indicates that the high-density areas in clay enrichment decrease after methane adsorption, whereas the low-density areas increase. As the adsorption pressure increases markedly, not all holes that are filled with clay minerals exhibit expansion deformation. The expansion deformation of clay minerals easily occur in areas of non-uniform density (in white line box area). It can be easily squeezed during expansion deformation because low-density areas exhibit strength, leading to density homogenization within the adjacent areas. For clay mineral structures to exhibit expansion deformation, two conditions are necessary: growth relative to pore structure and existing expansion space.

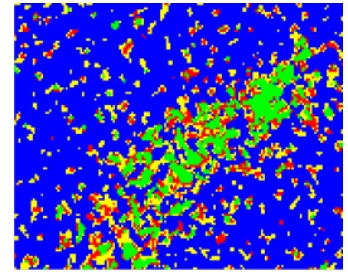

$1 \mathrm{MPa}$ CT pseudo color map

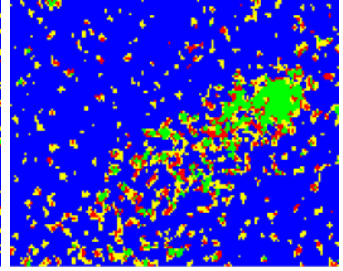

$5 \mathrm{MPa}$ CT pseudo color map

Fig. $8 \mathrm{CT}$ grayscale and pseudo-color image of pore development area filled with clay minerals

$$
\varepsilon=\frac{U V p}{1+V p}
$$

where $\varepsilon$ is the relative deformation value of the coal body; $p$ is the pore gas pressure in coal; $U$ denotes the maximum deformation value of coal after absorbing the gas; and $V$ is the parameter of the relationship between gas pressure and coal body expansion deformation. If the gas quality increased by adsorption is not considered, the expansion deformation value of 
the same area is proportional to the reduced density; therefore, the decreased density value also follows the Lundgren Muir equation:

$$
\Delta \rho=k \varepsilon=\frac{k U V p}{1+V p}
$$

where $\Delta \rho$ is the decrease in the value of coal density, and $k$ is the proportionality coefficient of expansion deformation, as well as decreased density value. Fitting for the B1, B2 and $\mathrm{C} 1, \mathrm{C} 2$ decreasing rate (Tables 2 and 3), and pore pressure Lundgren Muir equation, as well as the results, are shown in Table 4.

Table 4 Curve fitting of adoption and density decreasing rate of pore

\begin{tabular}{lcc}
\multicolumn{2}{c}{ development area } \\
\hline Area code & Fitting formula & $\begin{array}{c}\text { correlation } \\
\text { coefficient } / \mathrm{R}^{2}\end{array}$ \\
\hline
\end{tabular}

B1

$$
\Delta \rho=\frac{0.507228 \times 0.79889 p}{1+0.79889 p}
$$

0.9581

B2

$$
\Delta \rho=\frac{0.570093 \times 1.096038 p}{1+1.096038 p}
$$

0.9222

C1

$$
\Delta \rho=\frac{0.429886 \times 0.450003 p}{1+0.450003 p}
$$

0.8689

$\mathrm{C} 2$

$$
\Delta \rho=\frac{0.602482 \times 0.370706 p}{1+0.370706 p}
$$

0.923

The fitting results show that the decrease in the attenuation coefficient of the exhibiting pore structure development basically follows the Lundgren Muir equation. The equation indicates that the pore structure of the development area is the main methane adsorption area and the main location of coal expansion deformation. The parameter value of $V$ in the class B area is larger than that in the class $\mathrm{C}$ area, indicating that the unfilled pore structure exhibits stronger methane adsorption and deformation capacity than the pore structures filled with clay minerals.

\section{Pore fracture structure and clay mineral mixing} area

As shown in Fig. 2, the class D area is a mixed area with pore fissure structure and clay minerals. The upper left corner shows an obvious mesoscopic fracture in D1, and the center-right part is a large hole filled with clay minerals. D2 mainly includes vitrinite coal matrix, which contains some primary pores and different clay mineral holes exhibiting various shapes. The class D area displays several pore structures and clay minerals; thus, the inhomogeneity of the coal body structure is obvious. Under different adsorption pressures, the fluctuation of average attenuation coefficients (Fig. 9) widely ranges. The maximum fluctuation values of the average attenuation coefficient in D1 and D2 areas are 0.0014 and 0.0017, respectively. The pixel ratio with decreased attenuation coefficient in the Class D area under different adsorption pressures shows that (Table 5) the area average ratio with decreased attenuation coefficient in the class D area reaches $51.53 \%$ and $51.53 \%$, respectively. However, under different pressures, the fluctuation range of D1 and D2 volatility can reach $18.33 \%$ and $19.33 \%$ respectively. The areas with decreased density and the fluctuation range of overall density change in this region are very obvious. Studies ${ }^{[16]}$ indicate that mesoscopic damage occurs after methane adsorption and is mainly attributed to severe density changes under different adsorption pressures.

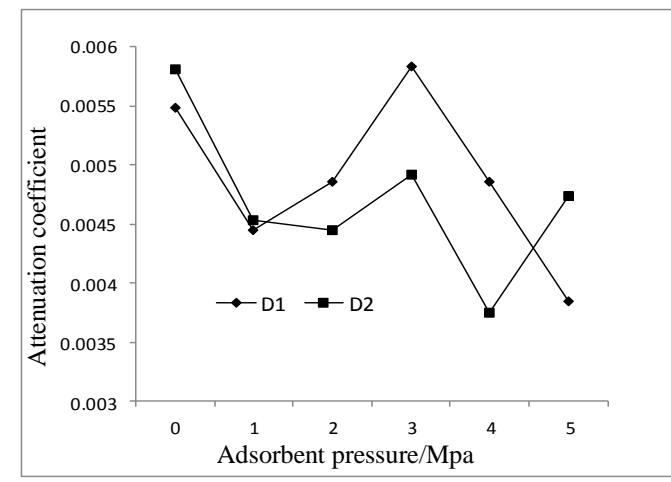

Fig. 9 Average attenuation coefficient changing curve with pore pressure change in the mixing area of pore fissure structure and clay minerals

Table 5 Ratio of the area exhibiting decreased density in the mixed region of pore fissure structure and clay minerals

\begin{tabular}{ccc}
\hline \multirow{2}{*}{$\begin{array}{c}\text { Adsorbent pressure } \\
\text { /MPa }\end{array}$} & \multicolumn{2}{c}{ Area code } \\
\cline { 2 - 3 } & $\mathrm{D} 1$ & $\mathrm{D} 2$ \\
\hline 1 & $50.33 \%$ & $66.00 \%$ \\
\hline 2 & $41.67 \%$ & $46.33 \%$ \\
3 & $52.00 \%$ & $56.67 \%$ \\
4 & $60.00 \%$ & $53.00 \%$ \\
5 & $53.67 \%$ & $49.33 \%$ \\
\hline
\end{tabular}

\section{Influence of methane adsorption on coal body density}

The coal mesoscopic structure changes after methane adsorption. In microscopic CT experiments, 
such variation represents the change in attenuation coefficient. The degree of change in attenuation coefficient reflects the different methane adsorption capacities and degrees of deformation among various coal body structures. The same also reflects the damage in the mesoscopic structure caused by methane adsorption.

During methane adsorption, the coal body density undergoes obvious changes. Analysis shows that the degree of change is related to adsorption pressure. As shown in Table 6, after methane adsorption, the average attenuation coefficient of the coal body decreases. As adsorption pressure increases, the average attenuation coefficient decreases more significantly.

Table 6 Change in attenuation coefficient under varying adsorption pressures

\begin{tabular}{cccccc}
\hline $\begin{array}{c}\text { Adsorbent } \\
\text { pressure/MPa }\end{array}$ & 1 & 2 & 3 & 4 & 5 \\
\hline $\begin{array}{c}\text { Average attenuation } \\
\text { coefficient }\end{array}$ & 0.00426 & 0.00419 & 0.00408 & 0.00386 & 0.00351 \\
$\begin{array}{c}\text { Absolute change } \\
\text { Reduced attenuation } \\
\text { coefficient range }\end{array}$ & $19.92 \%$ & $24.22 \%$ & $28.52 \%$ & $34.77 \%$ & $47.66 \%$ \\
\hline
\end{tabular}

As shown in Fig. 10, after methane adsorption, the change in coal attenuation coefficient distribution ratio presents a change in "sine curve" with the attenuation coefficient. After methane adsorption, the low-density areas increase, whereas the high-density areas decrease. The increases in attenuation coefficient mainly range from 0.015 to 0.045 , and the decreases in attenuation coefficient mainly range from 0.045 to 0.045 . With a decrease in the distribution ratio, the range of the attenuation coefficient increases first and then decreases; this behavior is followed by an increase again as adsorption pressure is increased (Table 6). At $1 \mathrm{MPa}$, the attenuation coefficient

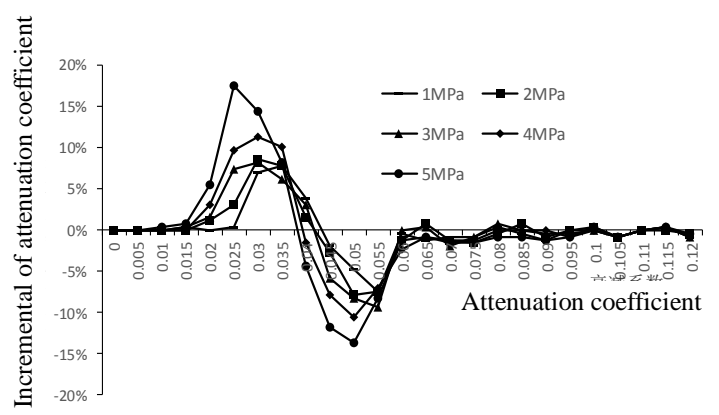

Fig. 10 Change in attenuation coefficient of coal samples under varying adsorption conditions distribution ratio is $19.92 \%$ of the change. With increasing adsorption pressure, the degree of change becomes increasingly obvious. At $5 \mathrm{MPa}$, the change in the total attenuation coefficient distribution ratio reaches $47.66 \%$. This result suggests that the coal body exhibits expansion deformation after methane adsorption, and the expansion of the heterogeneous media deformation is uniform. As adsorption pressure increases, the range of expansion deformation first decreases and then increases. The expansion deformation lead to an increase in the number of low-density areas and a decrease in the number of high-density areas. The degrees of expansion deformation degree become increasingly obvious, thereby decreasing the mechanical strength after methane adsorption.

The comparison of the concentration of density distributions in coal are shown in Table 7. The area within the scope of 0.02 to 0.06 increases as adsorption pressure increases from $89.06 \%$ to $95.7 \%$. In addition, $1.17 \%$ of the areas have an average attenuation coefficient of less than 0.02 , and the area with an average attenuation coefficient exceeding 0.06 is significantly reduced. These results are attributed to the tension of expansion deformation. These results also suggest that the concentration of coal density distribution increases, and the overall density of coal generally tends to exhibit homogenization. The main reason is that the area filled with high-density clay minerals exhibit expansion deformation after methane adsorption, leading to decreased density.

Table 7 Distribution rule changing with adsorption pressure within the scope of different attenuation coefficient

\begin{tabular}{cccc}
\hline \multirow{2}{*}{$\begin{array}{c}\text { Adsorbent pressure } \\
\text { /MPa }\end{array}$} & \multicolumn{3}{c}{ The range of attenuation coefficient } \\
\cline { 2 - 4 } & $<0.02$ & $0.02-0.06$ & $>0.06$ \\
\hline 0 & $0.00 \%$ & $89.06 \%$ & $10.94 \%$ \\
1 & $0.00 \%$ & $92.19 \%$ & $7.81 \%$ \\
2 & $0.39 \%$ & $92.19 \%$ & $7.42 \%$ \\
3 & $0.39 \%$ & $93.75 \%$ & $5.86 \%$ \\
4 & $0.78 \%$ & $94.14 \%$ & $5.08 \%$ \\
5 & $1.17 \%$ & $95.7 \%$ & $3.13 \%$ \\
\hline
\end{tabular}

\section{Deformation rule of coal methane adsorption}

Under different adsorption pressures, swelling capacity and mechanical strength vary because the adsorption rates of different coal body structures. Different areas of coal exert two effects: (1) expansion 
deformation after adsorption and (2) squeezing each other. When the effect of expansion deformation is greater than that of mutual extrusion, the average attenuation coefficient of coal is reduced; when the effect of expansion deformation is less than that of mutual extrusion, the average attenuation coefficient is increased.

\subsection{Expansion extrusion effect of coal methane adsorption}

Table 8 presents the results of the occupied area of attenuation coefficient variation under different adsorption pressure. The change in attenuation coefficient mainly ranges from 0.001 to 0.003 . The area of deformation is greater than the area of extrusion, and the ratio is about 5 to 12 times, which shows that the expansion effect of coal methane adsorption is stronger than its squeezing effect; a greater adsorption pressure indicates a more obvious occurrence. After methane adsorption, the axial direction occupies sufficient space, which can be attributed to the area on the surface of the coal body; therefore, the mutual extrusion effect is relatively weaker than the inside of coal. The density increasing area increases and then decreases as adsorption pressure increases; when the densities are at 1 and 2 $\mathrm{MPa}$, the density increasing areas reach $20.7 \%$ and $26.6 \%$, respectively. With a further increase in adsorption pressure, the density increasing area continues to decrease; when the adsorption pressure is $5 \mathrm{MPa}$, the density increase is only $7.4 \%$ because of the squeezing effect.

Table 8 Proportion and variance change of the attenuation coefficient under varying adsorption pressures

\begin{tabular}{|c|c|c|c|c|c|c|c|c|}
\hline \multirow{2}{*}{$\begin{array}{c}\text { Adsorbent pressure } \\
\text { /MPa } \\
\end{array}$} & \multicolumn{6}{|c|}{ Interval of attenuation coefficient } & \multirow{2}{*}{$\begin{array}{l}\text { Total amount of } \\
\text { reduction }\end{array}$} & \multirow{2}{*}{$\begin{array}{c}\text { Total amount of } \\
\text { increase }\end{array}$} \\
\hline & $>0.001$ & $(00.001)$ & $(-0.0010)$ & $(-0.002-0.001)$ & $(-0.002-0.003)$ & $<-0.003$ & & \\
\hline 1 & $0.4 \%$ & $20.3 \%$ & $53.9 \%$ & $23.4 \%$ & $1.6 \%$ & $0.4 \%$ & $79.3 \%$ & $20.7 \%$ \\
\hline 2 & $2.0 \%$ & $24.6 \%$ & $49.2 \%$ & $18.4 \%$ & $5.1 \%$ & $0.8 \%$ & $73.4 \%$ & $26.6 \%$ \\
\hline 3 & $0.8 \%$ & $18.4 \%$ & $48.0 \%$ & $26.6 \%$ & $5.5 \%$ & $0.8 \%$ & $80.9 \%$ & $19.1 \%$ \\
\hline 4 & $1.2 \%$ & $10.5 \%$ & $42.6 \%$ & $38.7 \%$ & $6.6 \%$ & $0.4 \%$ & $88.3 \%$ & $11.7 \%$ \\
\hline 5 & $1.2 \%$ & $6.3 \%$ & $30.1 \%$ & $42.6 \%$ & $15.6 \%$ & $4.3 \%$ & $92.6 \%$ & $7.4 \%$ \\
\hline
\end{tabular}

To explore the changing rule of the coal samples with different densities after methane adsorption, the distribution ratios within various attenuation coefficients for density increasing areas $(\Delta \mu>0)$ are determined, and the results are shown in Fig. 11. The attenuation coefficients within distribution range of density increasing areas are mainly distributed from 0.02 to 0.06 ; under adsorption pressure from $1 \mathrm{MPa}$ to $2 \mathrm{MPa}$, the increasing areas between 0.02 and 0.045 attenuation coefficients comprise $66.03 \%$ and $72.05 \%$. For adsorption pressure of $5 \mathrm{MPa}$, the increasing area

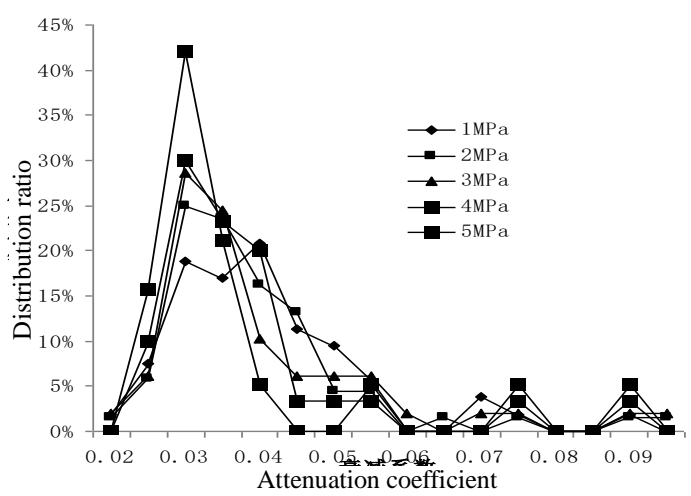

Fig. 11 Attenuation coefficient distribution diagram in increasing density area ranges from 0.02 to 0.04 , which comprise $84.21 \%$. The attenuation coefficient distribution tends to be concentrated with an increase in adsorption pressure.

The change in density change after the methane adsorption is associated with the original structure of the coal sample. In the low-pressure adsorption phase, the coal body exhibiting expansion deformation is more inclined to obtain an expansion space by squeezing original pore fissure of the coal structure; thus, the compression can change the primary pore fissure of coal and other defects within each density range. This change prompts a high-density area to expand and the attenuation coefficient distribution to widen. With an increase in pressure adsorption, the pore fracture structure emerges closure in the coal body, losing sufficient deformation space; the squeezing effect is concentrated in the original structure of the coal area with low density and strength.

Observation of the diagram of the change in fissure structure under low-pressure adsorption shows the effect of coal body expansion deformation on the fissure structure, as shown in Fig. 12. The electron 
microscope scanning image (A) and CT image (B) reveal a mesoscopic crack with a width of about $3 \mu \mathrm{m}$. After methane adsorption, the fracture position in the CT image is extruded, and the density increases (D); the mesoscopic fracture structure is almost completely closed after methane adsorption (C). The fissure

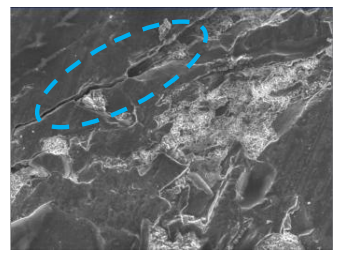

A SEM image of fracture

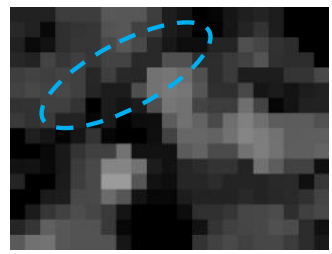

B 0Mpa CT image of fracture

structure is the main channel of methane migration in coal, and coal adsorption of methane with expansion deformation causes the original closure of fracture structure, which significantly affects the coal migration efficiency.

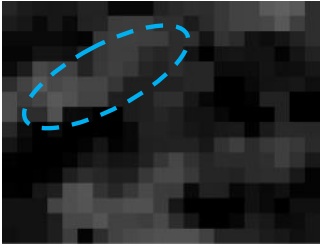

C 1 Mpa CT image of fracture

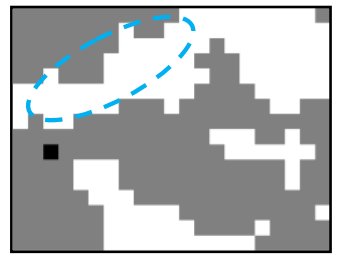

D changing CT image of fracture

Fig. 12 Variation trend of the fracture inside the coal after methane adsorption

\subsection{Expansion deformation rule of methane adsorption on coal}

To study the trend in expansion deformation, the slightly reduced $(-0.015<\Delta \mu<0)$ area and significantly decreased area $(\Delta \mu<-0.015)$ in different attenuation coefficient ranges statistics are evaluated. The statistical results are shown in Figs. 13 and Fig. 14 , respectively.

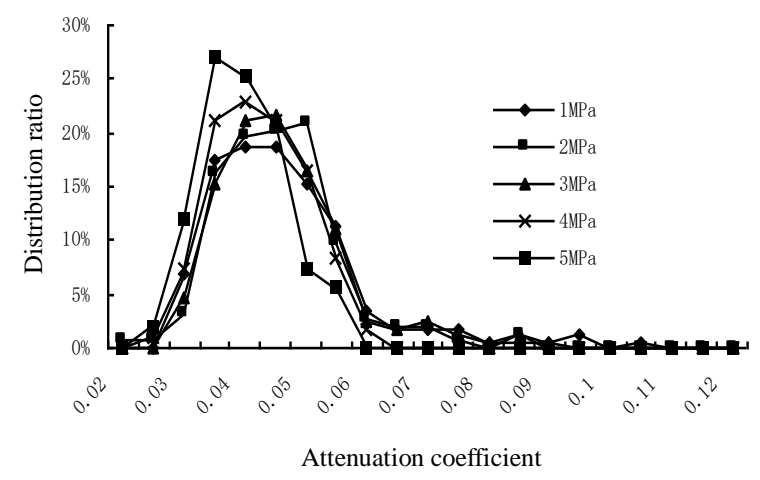

Fig. 13 Attenuation coefficient distribution in the density of slightlv reduced area

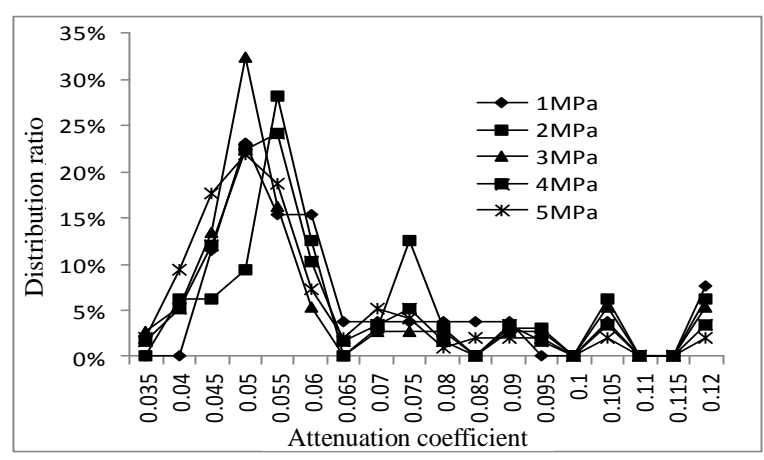

Fig. 14 Attenuation coefficient distribution in the density of obviously reduced area
Statistical results show that the attenuation distribution range with density slightly decreased after methane adsorption from 0.025 to 0.065 (above 90\%), which is similar to that of the increasing density area. The slightly decreased area becomes concentrated as adsorption pressure is increased. At a pressure adsorption of $1 \mathrm{MPa}$, the attenuation coefficient in the slightly reduced area ranges from 0.025 to 0.055 , comprising $76.84 \%$ of the total; at an adsorption pressure of $5 \mathrm{MPa}$, the slightly reduced area within the same attenuation coefficient is as high as $94.39 \%$. The significantly reduced area is distributed between 0.035 and 0.1 (above 90\%); with an increase in adsorption pressure, its distribution law exhibits no obvious change.

Averaging the distribution ratio (Fig. 15) under varying adsorption pressure reveals that extrusion causes the attenuation coefficient increasing area to obtain the lowest average density (0.035); expansion deformation prompts the attenuation coefficient slightly decreasing are the second average density

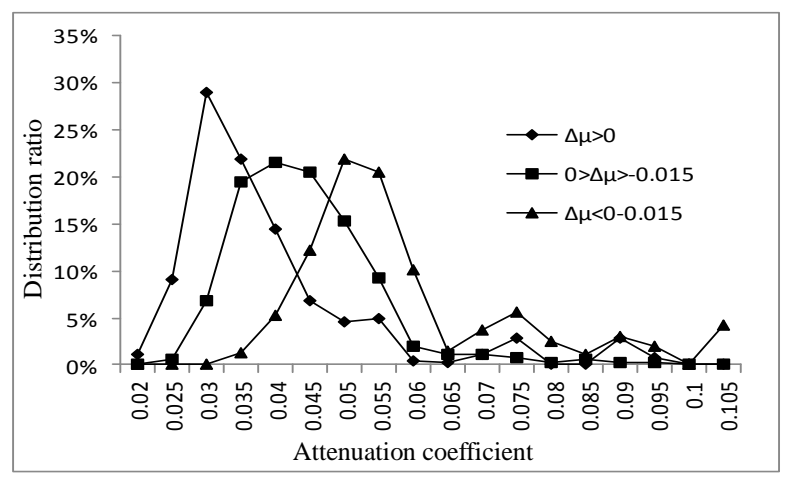

Fig. 15 Attenuation coefficient distribution ratios of different changing trends 
(0.045) and the attenuation coefficient of the remarkably reduced area has the biggest average density (0.055). It suggests that after methane adsorption, the higher the density of the coal area, the smaller the increase in low density area. In addition, with an increase in adsorption pressure, expansion /deformation of the internal structure of coal occurs from high-density to low-density areas.

\section{Conclusion}

SEM images reflect intuitively the microscopic structure of coal, and CT scanning can accurately measure the various physical parameters of coal under different adsorption states. By combining SEM and CT scanning, the following conclusions regarding the characteristics of deformation after methane adsorption on coal can be drawn:

1) SEM and CT scanning can exhibit a satisfactory relationship. By contrast, coal is a natural rock and most of attenuation coefficients fall within a narrow range. The coal surface consists of vitrinite coal matrix and clay minerals; in addition, it exists in the complex pore fissure structure. Comparison of SEM images indicates that the gray part with a lower density consists of vitrinite and a small amount of clay mineral structures. The large-density bright white clusters or spots are mainly composed of pore structures filled with clay minerals.

2) After methane adsorption, coal exhibits non-uniform expansion deformation, leading to a reduction in the low-density area and an increase in the high-density area. A higher adsorption pressure indicates a more obvious expansion deformation. The density range of the expansion deformation decreases and then increases. The coal density decreases, and density distribution becomes more concentrated because of the expansion deformation of the clay minerals.

3) During adsorption expansion, the opposite effects of mutual extrusion and expansion deformation occur within the coal structure. In addition, the effect of expansion deformation is stronger than that of mutual extrusion. In low-pressure adsorption phase, the coal expansion deformation is more inclined to first squeeze the original pore fissure of the coal structure to obtain an expansion space. After the adsorption pressure is increased, the effect of extrusion is mainly concentrated in the low-density area. With an increase in adsorption pressure, expansion deformation occurs from the high-density area to the low-density area.

4) Methane adsorption and expansion deformation of coal are related to the pore structures of coal. Adsorption and expansion mainly occur in the unfilled area or the pore structure area filled with clay minerals Expansion deformation complies with the Lundgren Muir equation. When the structure of clay minerals in the coal body occurs during expansion deformation, pore structure and expansion space are needed for development. Almost no adsorption expansion occurs in the area where the pore structure fails to develop. The area with both pore fissure structure and clay minerals obtains a large degree and range of deformation.

\section{Acknowledgments}

The authors are grateful to the National Science Foundation of China for financial support (21373146, 51304142) and Fundamental research project of Shanxi in China (2013021029-3)

\section{References}

ASTM E1935-1997(Reapproved 2003), Standard Test Method for Calibrating and Measuring CT Density.

Cetine,Gupatal,Moghtaderib. 2004, Effect of pyrolysis pressure and heating rate on radiate pine char structure and apparent gasification reactivity. Fuel, 83:1469-1482.

Feng Zengchao. Theoretical and application of strength exploitation on low percolation coalbed. Beijing: China Science Press,2008( in Chinese).

He X., Wang En., Lin H., 1996, Coal deformation and fracture mechanism under pore gas action. Journal of China University of Mining and Technology, 25(1):6-10( in Chinese).

Ji L., Qiu J., Xia Y., Zhang T., 2012, Micro-pore characteristics and methane adsorption properties and methane adsorption properties of common clay minerals by electron microscope scanning. Acta petrolei sinica, 33(2):249-256( in Chinese).

Karacan C.O. and Okandan E. 2001, Adsorption and gas transport in coal microstructure: investigation and evaluation by quantitative X-ray CT imaging. Fuel, 80:509-520.

Li Y., Li X., Li K., 1999, Using computed tomography to determine the basic petrophysical properties of cores, Petroleum exploration and development, 26(6):86-90( in Chinese).

Lin B., Zhou Sh., 1986, Experimental investigation on the deformation law of coal body contationing methine. Journal of China University of 
Mining Technology, 3:9-16( in Chinese).

Meng Y., Tang D., Xu H., Qu Y., Li Y., Zhang W., 2014, Division of coalbed methane desorption stages and its significance. Petroleum Exploration and Development, 41(5):671-677

Pan Zh., Luke D. Connell, 2007, A theoretical model for gas adsorption-induced coal swelling. International Journal of Coal Geology. 69:243-252

Ramesh K Sharma, Jan B Wooten, Vicki L. Baliga, Xuehao Lin, W. Geoffrey Chan, Mohammad R. Hajaligol, 2004, Characterization of chars from pyrolysis of lignin. Fuel, 83(11-12):1328-1482.

Wu Sh., Zhao W., 2005, Analysis of effective stress in adsorbed methane-coal system, Chinese Journal of Rock Mechanics and Engineering, 24(10):1674-1678( in Chinese).
Wang En., He X., Lin H., 1996, States of methane in coal deposit. Coal Engineer, 5:12-19( in Chinese).

Yu Y., Hu Y., Liang W., Meng Q., Feng Z., Yu Ch., 2010,Micro-CT experimental research of lean coal thermal cracking laws. Journal of China Coal Society, 35(10):1696-1700( in Chinese).

Zhang H., Li X., 2004, The application of SEM in coal petrology. Journal of Chinese Electron Microscopy Society, 23(4):467-468( in Chinese).

Zhao Y., Li X., Lu Y., Kang Y., 2009, Influence of gas pressure on compressive strength size effect of inhomogenous coal. Journal of China Coal Society, 34(8) : 1081-1085( in Chinese).

Zhou Sh., Lin B., 1990, The theory of gas flow and storage in coal seams. BeiJing: China Coal Industry Publishing House( in Chinese). 